
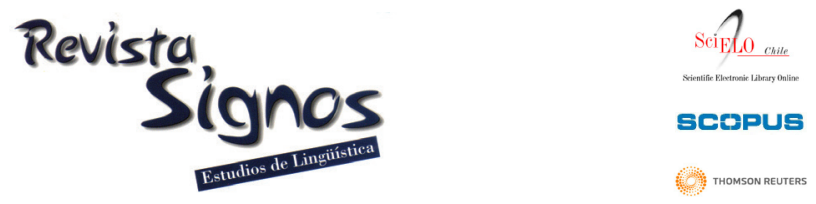

\title{
Aplicación de un programa de estimulación de la conciencia fonológica en preescolares de nivel transición 2 y alumnos de primer año básico pertenecientes a escuelas vulnerables de la Provincia de Concepción, Chile ${ }^{1}$
}

\section{Application of stimulation Program of Phonological Awareness in Pre-School children and First Year Elementary School students, who attend socially vulnerable schools in Concepción, Chile}

Beatriz Arancibia

Universidad Católica de la Santísima Concepción Chile

barancib@ucsc.cl
Marcela Bizama

Universidad Católica de la Santísima Concepción Chile mbizama@ucsc.cl

\section{Katia Sáez \\ UNIVERSIDAd DE CONCEPCIÓN \\ Chile \\ ksaez@udec.cl}

Recibido: 15-III-2011 / Aceptado: 3-IV-2012

\section{Resumen}

La conciencia fonológica (CF) es la habilidad para acceder conscientemente a la estructura de la lengua oral, lo que implica reconocer y manipular los segmentos fonológicos del habla. Numerosos estudios muestran la importancia de la CF para el éxito en el aprendizaje de la lectura, particularmente en sistemas alfabéticos como el español. Por lo tanto, es fundamental propiciar el desarrollo de las habilidades metafonológicas en niños prelectores y en la etapa de lectura inicial. El presente artículo presenta resultados parciales de una investigación aplicada cuyo objetivo fue pilotear un programa destinado a estimular el desarrollo de la conciencia fonológica en 20 preescolares de nivel transición 2 y 18 escolares de 1er año básico pertenecientes a escuelas vulnerables de la provincia de Concepción. La parte del estudio de la cual se 
informa corresponde a un diseño cuasi experimental, con pre y postest basados en la aplicación de la Prueba de Segmentación Lingüística (PSL) de Orellana y Ramaciotti (2007), estandarizada para la población chilena. El tratamiento consistió en el desarrollo de 15 sesiones de trabajo con cada grupo participante. Los resultados muestran efectos positivos de la intervención y confirman algunos aspectos del desarrollo de la conciencia fonológica señalados en la literatura. Se concluye que es posible mejorar las habilidades metafonológicas de los niños a través del programa diseñado, el cual puede ser mejorado incorporando aspectos no contemplados inicialmente.

Palabras Clave: Conciencia fonológica, intervención, preescolares, primer año básico.

\begin{abstract}
Phonological awareness is the skill to access consciously to the structure of the spoken language. This implies recognizing and manipulating the phonological segments of spoken language. Numerous researches show that phonological awareness is critical in learning to read in alphabetic languages like Spanish. Therefore, stimulating the methaphonological skills development in pre-readers children and children who are beginning to read is very important. This article presents some of the results of an applied investigation designed to test a program to develop phonological awareness in 20 pre-school children and 18 first year elementary school students, who attend socially vulnerable schools in Concepción, Chile. The part of the study which is reported corresponds to a quasi-experimental design with pre and post test based on Linguistic Segmentation Test (LST) by Orellana y Ramaciotti (2007), standardized for Chilean people. Treatment consisted in the development of 15 sessions with each participating group, using material specially designed by the researchers to train different aspects of phonological awareness. The results show positive effects of the intervention and confirm some aspects of the development of phonological awareness found in the literature. It concludes that it is possible to improve the children's methaphonological skills through the designed programme, which can be improved incorporating aspects that were not considerated originally.
\end{abstract}

Key Words: Phonological awareness, treatment, pre-school children, first year elementary school. 


\section{INTRODUCCIÓN}

La conciencia fonológica, es decir, la habilidad para manejar conscientemente los segmentos sonoros de la lengua (Treiman, 1991; Jiménez, 2009), es considerada actualmente como uno de los factores que mejor predice el aprendizaje de la lectura: dificultades en tareas que requieren conciencia fonológica (CF), especialmente a nivel de conciencia silábica en niños prelectores y conciencia fonémica en quienes han recibido instrucción lectora, se relacionan con mayores dificultades o retraso en el aprendizaje de la lectura. En Chile, Bravo Valdivieso, Villalón y Orellana (2006) confirman esas relaciones causales en niños de primer año básico (para una revisión, véanse Bravo Valdivieso, 2002, 2006; Márquez \& de la Osa, 2003; Herrera \& Defior, 2005).

La relación entre CF y lectura es particularmente importante en lenguas transparentes como el español, en que los niños tienen que adquirir el principio alfabético para aprender a leer, principio según el cual formas sonoras diferentes se representan con letras diferentes, y formas sonoras iguales se representan con letras iguales. Esto quiere decir que para lograr establecer la relación grafema-fonema y reconocer el sonido resultante de la combinación de grafemas, el niño tiene que discriminar entre las letras, atribuirles un sonido y sintetizar dos o más fonemas para leer sílabas y palabras (Jiménez \& Ortiz, 1995).

No se trata de que los niños sean plenamente conscientes de la estructura sonora de su lengua antes de aprender el lenguaje escrito, pero sí de que hayan alcanzado un nivel mínimo de CF que les permita hacer explícitas las reglas de correspondencia grafema-fonema y, por consiguiente, les resulte más fácil descomponer el código ortográfico (Porta, 2008). Jiménez (2009) señala que un nivel mínimo de CF para adquirir las habilidades lectoras básicas proporcionaría a su vez un soporte para el desempeño en tareas fonológicas más complejas. Por su parte, Bravo Valdivieso (2002, 2006) plantea que el nivel de desarrollo de la CF al inicio del aprendizaje de la lectura representa, siguiendo a Vigotzky (1988) una 'zona de desarrollo próximo' (ZDP) o indicador de la distancia entre el lenguaje oral del niño y su aprendizaje inicial de la lectura.

Teniendo en cuenta la importancia de la CF en el aprendizaje de la lectura, nos propusimos elaborar un programa psicopedagógico para desarrollar esta habilidad en niños de nivel transición 2 y en escolares de primer año básico. El supuesto central de base es que mediante la aplicación sistemática de un programa de estimulación de la $\mathrm{CF}$, niños y niñas pueden mejorar significativamente sus habilidades metafonológicas.

En el presente trabajo, se informa de los resultados obtenidos en una aplicación piloto destinada a poner a prueba parte de los materiales diseñados. El objetivo es probar la efectividad de las actividades diseñadas mediante su implementación en aula. Para ello, se recurrió en esta oportunidad a un diseño de investigación cuasi experimental con pre y postest, aplicado a una muestra de niños pertenecientes al 
estrato socioeconómico bajo, condición que no solo está asociada a un desempeño escolar más bajo en lectura ${ }^{2}$, sino también a un menor desarrollo lingüístico en comparación con el estrato socioeconómico alto (Pavez, Maggiolo, Peñaloza \& Coloma, 2009).

\section{Marco conceptual}

\subsection{Niveles de conciencia fonológica}

La CF considera diferentes componentes con distintos niveles de complejidad, según cual sea la unidad lingüística objeto de reflexión y manipulación por parte del sujeto y los procesos que realice sobre ella. En la literatura es posible encontrar distintos criterios para señalar los niveles de segmentación fonológica. Treiman (1991) propuso un modelo jerárquico y acotado, ampliamente aceptado, que incluye tres componentes o niveles: la conciencia silábica, que corresponde a la habilidad para segmentar, identificar o manipular conscientemente las sílabas que componen una palabra; la conciencia intrasilábica, que corresponde a la habilidad para segmentar las sílabas en sus componentes intrasilábicos de onset y rima; la conciencia fonémica, vale decir, la capacidad para darse cuenta de que las palabras habladas están constituidas por unidades sonoras discretas o unidades mínimas no significativas, los fonemas.

En todos los niveles, la habilidad de segmentación le permite al niño comprender las relaciones existentes entre lenguaje escrito y lenguaje hablado, y le facilita el proceso de descodificación y codificación cuando comienza a leer. No obstante, utilizar un nivel de segmentación u otro depende de las características de cada lengua en particular, pues ellas constriñen el tipo de análisis que los niños pueden hacer. Mientras en lenguas como el inglés la memorización y la rima les son útiles para establecer analogías que les facilitan el reconocimiento de palabras, en lenguas con ortografía transparente, como el español, los niños pueden recurrir a sus conocimientos sobre la correspondencia grafo-fonética. Por lo tanto, resulta fundamental el conocimiento explícito por parte del niño de que las palabras están formadas por una cadena de unidades fonológicas que pueden ser articuladas por sí mismas, las sílabas, y que -a su vez- estas se pueden descomponer en los sonidos que las conforman (Jiménez, 1992).

Las tareas mediante las cuales se evalúa el desarrollo de los distintos componentes de la CF también difieren en cuanto a su complejidad, según los procesos cognitivos que ellas involucran. Así, mientras algunas tareas requieren una sola operación por parte del sujeto, como puede ser identificar el sonido inicial de una palabra, otras requieren dos, como ocurre con la síntesis fonémica. En este último caso, el coste cognitivo es mayor, pues se debe mantener la serie de fonemas como estímulo activo en el retén fonológico de la memoria de trabajo verbal para poder componer la palabra que la serie forma. Al respecto, diversos estudios muestran que la capacidad de memoria verbal de corto plazo se relaciona con la CF y es un factor que también 
predice la adquisición lectora (Mann \& Liberman, 1984; Gathercole \& Baddeley, 1993; Scanlon \& Vellutino, 1997; McCallum, Bell, Wood, Below, Choate \& McCane, 2006). En particular, en un estudio previo cuya muestra incluyó a los participantes de esta investigación, Bizama, Arancibia y Sáez (2011), encontraron una correlación positiva entre memoria verbal y segmentación fonológica, tanto en niños de nivel transición 2 como de primer año básico.

Una propuesta que contribuye a clarificar las diferencias entre las tareas con que se evalúa la CF es la de Bravo Valdivieso, Villalón y Orellana (2002), quienes distinguen entre 'tareas pasivas' y ‘tareas activas'. Entre las primeras están las de identificación (vg. identificar la sílaba inicial de una palabra), que serían las más sencillas; la segundas, en cambio, implican intervención sobre las palabras (por ejemplo, síntesis de fonemas, omisión de sílaba) y serían más complejas.

\subsection{Desarrollo de la conciencia fonológica}

Desde el punto de vista evolutivo, la CF se desarrolla fuertemente durante el período comprendido entre los 4 y 8 años de edad y tiende a seguir un curso que va desde la conciencia silábica, hasta culminar con el manejo de habilidades fonémicas una vez que los niños aprenden a leer y a escribir (Anthony \& Francis, 2005). Jiménez y Ortiz (1993) muestran que el mayor incremento se produce entre el segundo nivel de jardín infantil y el segundo curso de enseñanza básica, lo que se relaciona con los procesos de la lecto-escritura. Al respecto, investigaciones recientes sugieren que la relación entre la CF y dichos procesos es bidireccional y recíproca: la CF apoya y favorece la adquisición de la lecto-escritura, y el aprendizaje de esta favorece igualmente la CF (Jiménez, García \& Venegas 2010).

El desarrollo de la CF involucra tanto un aumento en la toma de conciencia sobre las unidades fonológicas del lenguaje hablado, como un incremento de la capacidad para manipularlas (Treiman, 1991; Gillam \& van Kleeck, 1996; Bravo Valdivieso, 2006). Las unidades que parecen ser más relevantes son la sílaba y el fonema, aunque existe acuerdo en que la sílaba es la unidad que los niños pueden reconocer y manipular con mayor facilidad, aún antes de aprender a leer (Liberman, Shankweiler, Fischer \& Carter, 1974; Fox \& Routh, 1975; Manrique \& Gramigna, 1984; Jiménez, 1992; Jiménez \& Ortiz, 2000). En español, la conciencia silábica también parece ser la más accesible. Jiménez y Ortiz (1995), en un estudio longitudinal con niños españoles, encontraron que la segmentación silábica era más fácil que la segmentación fonémica en el inicio lector. No obstante, en el nivel silábico, también hallaron diferencias asociadas al tipo de tarea, pues a los niños les resultaba más fácil segmentar palabras en sus sílabas constituyentes que omitir sílabas, lo que alude a la ya señalada diferencia entre 'tareas activas' versus 'pasivas'.

La explicación más común para dar cuenta de la predominancia de la conciencia silábica, es que la sílaba, como unidad básica de articulación, es menos abstracta que 
los fonemas, lo que facilita la toma de conciencia de su existencia en el lenguaje oral y el consecuente desarrollo de la habilidad para segmentar silábicamente las palabras. Además, en español, las regularidades de la estructura silábica facilitan la identificación de las sílabas en el lenguaje escrito (Carrillo \& Marín, 1996).

Varias investigaciones (Mann \& Liberman, 1984; Rueda, Sánchez \& González, 1990; Carrillo \& Sánchez, 1991) también dan cuenta del papel facilitador de la conciencia silábica en el aprendizaje de la lectura, por su relación con la facilidad y rapidez para aprender las habilidades básicas de descodificación de palabras. Sin embargo, estudios longitudinales con niños españoles muestran que el poder predictivo de la conciencia silábica tiende a disminuir cuando los escolares ya han comenzado a leer, cediendo su lugar a la conciencia fonémica (Carrillo, 1994).

Los fonemas son unidades más pequeñas y abstractas que la sílaba, y son particularmente difíciles en niños no lectores (Jiménez \& Ortiz, 1995). Por esto, el desarrollo de habilidades que implican conciencia intrasilábica y, especialmente, fonémica, es un poco más tardío y requiere entrenamiento especial (Treiman, 1991; Rueda, 1995). Al respecto, aunque el aprendizaje de la lectura en un sistema alfabético resulta crucial para el desarrollo de la conciencia fonémica debido a la ejercitación del principio alfabético, manipular los sonidos accediendo a ciertos niveles de conciencia fonémica es al mismo tiempo condición necesaria para la adquisición del principio alfabético (Cuadro \& Trías, 2008). En este sentido, las evidencias son claras en cuanto a respaldar la idea de que un déficit en conciencia fonémica en el inicio lector es el predictor más potente de retraso lector en una ortografía transparente (Jiménez, 1996).

En Chile, Bravo Valdivieso et al. (2001, 2002, 2003a, 2003b, 2006) han encontrado que la conciencia fonémica es el aspecto de la CF que mejor predice el aprendizaje de la lectura y escritura hasta el tercer año de escolaridad: los niños capaces de identificar y aislar los fonemas de una palabra, tienen mejor base cognitiva para asociar los sonidos de las palabras escritas con su correspondiente grafía. Resultados similares se han encontrado en estudios longitudinales con niños de habla inglesa, en los que la conciencia fonémica se ha comportado como un mejor predictor de la lectura que las habilidades para identificar unidades mayores como el onset y la rima entre los 5 y 6 años de edad (Hulme, Hatcher, Nation, Brown, Adams \& Stuart, 2002).

Considerando todo lo anterior, en el diseño de actividades para el desarrollo de la CF hay que tener en cuenta los distintos grados de dificultad de las tareas que se solicitan. Para ello es preciso considerar sus demandas cognitivas y el tipo de unidad lingüística que los niños pueden manipular de acuerdo con su edad y experiencia con la lengua escrita (Cuadro \& Trías, 2008). En la etapa de 4-5 años es recomendable realizar actividades de segmentación léxica y silábica, para, posteriormente, en el último nivel de educación preescolar y durante todo el primer año de educación básica, incorporar la segmentación fonémica, pues el desarrollo de las habilidades 
de segmentación intrasilábica no solo se relaciona con la edad, sino también con la experiencia que el niño va adquiriendo con el material escrito al comienzo de su inmersión en los procesos de lecto-escritura. Además, es recomendable comenzar con tareas de análisis y después introducir tareas de síntesis, que implican un mayor nivel de abstracción.

\subsection{Entrenamiento en CF. Algunos antecedentes}

Una revisión de programas de estimulación de habilidades metalingüísticas para niños de habla hispana desarrollados en la última década, muestra que España lidera la producción. Tal vez el más conocido es el Programa de Entrenamiento en Conciencia Fonológica (PECONFO) de Jiménez y Ortiz (2007). Se trata de un programa de aplicación individual, que tiene como base empírica investigaciones realizadas en Canarias con niños prelectores. Se caracteriza por tener una secuencia de presentación de las tareas de complejidad creciente respecto de las unidades fonológicas objetos de reflexión: palabras, sílabas, unidades intrasilábicas y fonemas. Incluye una variedad de actividades, entre ellas el conteo, adición e inversión de sílaba o fonema, segmentación y síntesis.

Otro programa español para el desarrollo del conocimiento fonológico es Komunica (De la Torre, Guerrero, Conde \& Claros, 2002), orientado al trabajo en el aula con niños de jardín infantil y primer ciclo de educación básica de habla andaluza. Contempla 62 fichas de trabajo, juegos de lenguaje y sugerencias de evaluación, que pueden ser aplicados de manera flexible.

Arnáiz, Castejón, Ruiz y Guirao (2002) desarrollaron un trabajo de campo cuyo objetivo fue comprobar la influencia de una enseñanza sistemática de habilidades fonológicas en el acceso a la lectura y escritura. Para ello, diseñaron un programa de desarrollo de habilidades fonológicas en los ámbitos léxico, silábico y fonémico, el cual fue implementado durante dos años a 39 niños de segundo ciclo de Educación Infantil de la Región de Murcia. Las actividades incluían la composición de palabras y la identificación de grafemas. En la evaluación final, estos niños obtuvieron mejores puntuaciones que el grupo control en habilidades fonológicas, produciéndose, asimismo, un efecto positivo y facilitador para el acceso y adquisición de la lectura y escritura en estos alumnos.

En Latinoamérica, una investigación interesante es la de Cuadro y Trías (2008) en la que aplicaron experimentalmente un programa focalizado en conciencia fonémica, en 51 niños uruguayos. El programa está orientado a preescolares de 5 a 6 años de edad y diseñado para ser aplicado en 16 sesiones de 30 minutos cada una. Las distintas tareas propuestas son: aislamiento, reconocimiento, segmentación, síntesis, adición, omisión y sustitución de fonemas. Estas se presentan en un Programa Combinado en el cual se trabaja con el fonema y su grafema correspondiente de manera explícita y un Programa Simple, en el que se presenta solo el fonema. Los resultados mostraron 
efectos positivos del entrenamiento, especialmente en los niños que habían participado del programa combinado.

En Argentina, Rincón y Pérez (2009) diseñaron el PECF, un programa de entrenamiento de la conciencia fonológica concebido como un instrumento de dominio fonoaudiológico dirigido a preescolares de 5 años de edad. Los autores informan que su principal característica es que está basado en el juego como estrategia de motivación. Incluye el reconocimiento de grafemas y diversas tareas de conciencia fonémica y silábica, como la segmentación, aislamiento, omisión, sustitución y síntesis. Un aspecto interesante es que propone actividades de integración de las distintas habilidades y de evaluación de los logros que el niño va alcanzando. El programa fue sometido a validación mediante juicio de expertos.

En Perú, Velarde (2010) aplicó experimentalmente durante 6 meses, a razón de 3 sesiones semanales de 1 hora, el programa Jugando con los Sonidos en niños de 8 a 10 años de edad, que cursaban el tercer y cuarto grado de primaria, de nivel socioeconómico bajo, con el fin de probar sus efectos en descodificación lectora y la comprensión de la lectura. El programa, que solo se describe en términos generales, estaba destinado a estimular las habilidades de conciencia fonológica en los niveles de rima, sílaba y fonema en distintas estructuras lingüísticas y a través de diferentes tareas. El grupo experimental mejoró significativamente en todas las áreas de la CF evaluadas, tanto globalmente como en cada una de las habilidades trabajadas: síntesis, aislar, segmentar y omitir. Igualmente, se observó una mejora en la decodificación y comprensión lectora.

En nuestro país, la Fundación Astoreca cuenta con un Manual de Lenguaje para kínder (2007) para su empleo en los establecimientos educacionales que administra. Contempla actividades grupales e individuales de conciencia silábica y fonémica, graduadas según su complejidad. Incorpora sugerencias y orientaciones para su aplicación, indicaciones sobre el tiempo de duración de las tareas, explicaciones sobre los materiales requeridos y una ficha tipo glosario para el profesor. No se dispone de antecedentes relativos a alguna aplicación experimental para probar su efectividad.

Como se señaló al inicio, el presente trabajo se enmarca en un proyecto que tiene como objetivo el diseño de un programa para estimular el desarrollo de la CF en niños prelectores y en escolares que comienzan su aprendizaje de la lectura. La perspectiva desde la cual se ha enfocado el trabajo es el desarrollo de la CF como factor que favorece la adquisición del principio alfabético con que opera el aprendizaje lector. Consideramos que el papel de la investigación aplicada es fundamental para generar programas con sustento teórico y respaldo empírico. En este sentido, la mirada conjunta de la psicolingüística aplicada a los procesos básicos de la lectura y la psicopedagogía como disciplina de base para el diagnóstico, la intervención y la prevención de las dificultades de aprendizaje de la lectura representa un aporte para el trabajo sistemático en el desarrollo de habilidades metalingüísticas en educación inicial. 


\section{Metodología}

\subsection{Participantes}

Los participantes de la intervención implementada fueron 20 preescolares de nivel transición 2, pertenecientes a una escuela de párvulos municipal de la comuna de Concepción y 18 escolares de primer año básico, pertenecientes a otra escuela municipal de la misma comuna. La selección de la muestra fue intencionada, pues ambos establecimientos reciben a menores socialmente vulnerables y se emplazan en el mismo sector de la comuna, por lo que muchos de los niños que asisten a la escuela de párvulos prosiguen sus estudios de enseñanza básica en la escuela seleccionada. Un antecedente importante al respecto, y que fue considerado al momento de escoger la muestra, es que dicho establecimiento ha presentado sistemáticamente bajos puntajes en la prueba SIMCE de Lenguaje aplicada a los niños de cuarto año básico, ubicándose entre los más disminuidos de la provincia. Los datos referidos a la edad de los participantes se pueden apreciar en la Tabla 1.

Tabla 1. Distribución de la muestra según curso y edad.

\begin{tabular}{|c|c|c|c|c|c|}
\hline Curso & N & Media & D.E. & Min. & Máx. \\
\hline Nivel Transición 2 & 20 & 5 años 5 meses & 3 meses & 5 años & 6 años 1 mes \\
\hline Primer Año Básico & 18 & 6 años 4 meses & 3 meses & 6 años & 7 años \\
\hline
\end{tabular}

Para controlar variables no deseadas a nivel intrasujeto, se dejó fuera del estudio -aunque estuvieron presentes en las sesiones de trabajo- a los niños que formaban parte del programa de integración escolar, a quienes presentaban repitencia en primer año y a los que sobrepasaban el límite de edad para el nivel escolar. Además, se aplicó en forma colectiva el subtest de discriminación auditiva de la Prueba de Funciones Cognitivas Básicas (Berdicewski \& Milicic, 2002). Este test tiene como propósito evaluar en niños de entre 5 y 7 años la capacidad para percibir estímulos auditivos de tono bajo, discriminar sonidos sin claves visuales, reconocer sonidos iniciales y finales de palabras, y distinguir palabras según su duración. Los resultados mostraron que tanto los participantes de nivel transición 2 como los de primer año básico pertenecían al grupo de niños que sobrepasa el límite de lo esperado para su edad en discriminación auditiva, por lo que se consideró que no habría ingerencia de dificultades para diferenciar los sonidos durante la realización de las tareas de la CF.

\subsection{Instrumento empleado para evaluar la CF}

Para evaluar la CF, se utilizó la Prueba de Segmentación Lingüística, PSL, (Orellana \& Ramaciotti, 2007), instrumento validado y estandarizado para la población escolar chilena y que presenta una confiabilidad de 0.9564 estimada con el coeficiente de Kuder Richardson 20. La prueba evalúa la conciencia de la estructura segmental de la lengua en niños de 5 a 7 años en tres niveles: léxico, silábico y fonémico. Consta 
de 42 ítems agrupados en tres subtests. El primero incluye 8 ítems de segmentación de frases en palabras; el segundo consiste en dos tareas de conciencia silábica: segmentación silábica (8 ítems) e inclusión de sílaba en posición central de palabra (8 ítems). El tercer subtest evalúa conciencia fonémica mediante tres tareas: aislamiento del fonema inicial, síntesis y análisis de fonemas, con 6 ensayos cada una. A los niños de 6 y 7 años se les aplica la prueba completa. Los niños de 5 años solo realizan el subtest de segmentación silábica.

No se calcula un puntaje global, sino los puntajes directos por nivel, los que luego se llevan a una tabla estándar para su conversión en puntaje T según la edad y NSE (en este caso, 5 y 6 años de NSE bajo). Se trabaja así con una escala estandarizada que va de 20 a 80 puntos, con un promedio de 50 y una desviación estándar igual a 10 . Permite interpretar, en cada uno de los aspectos de la CF evaluados, si el desempeño de los niños se encuentra dentro (41-60), bajo (<41) o sobre $(>61)$ el desempeño estándar establecido para su edad y NSE por el instrumento utilizado. Por lo tanto, también es posible interpretar si el promedio alcanzado se aproxima al límite inferior o al límite superior dentro de cada rango.

\subsection{Procedimientos}

\subsubsection{Aplicación de pre y postest}

Las evaluaciones, que estuvieron a cargo de las investigadoras y un grupo de asistentes, se aplicaron en los mismos establecimientos y durante la jornada escolar habitual. Primero se aplicó la prueba de discriminación auditiva. Posteriormente, en una sala especialmente acondicionada al interior de cada establecimiento, se continuó con la aplicación de la PSL en forma individual, la cual se reaplicó en el postest en las mismas condiciones.

\subsubsection{Descripción de la intervención}

La intervención se llevó a cabo en 15 sesiones de trabajo desarrolladas en el aula habitual de cada grupo, con una duración aproximada de 30 minutos y una frecuencia de dos sesiones por semana. Todo el programa se efectuó dentro de la jornada escolar y estuvo a cargo de las investigadoras responsables y el equipo de asistentes, que fue previamente capacitado.

El programa consideró ejercicios agrupados en 3 áreas o componentes de la CF, de distintos niveles de complejidad:

a. Ejercicios de conciencia léxica: reconocimiento de palabras en el contexto de frases de dos y tres palabras; segmentación de frases contando sus palabras constituyentes; comparación de frases según su número de palabras; identificación de palabra agregada. 
b. Ejercicios de conciencia silábica: identificación, segmentación y manipulación de sílabas en palabras de distintas longitud y estructura silábica; comparación de palabras cortas y largas según el número de sílabas; emparejamiento de palabras por su sílaba inicial y sílaba final.

c. Ejercicios de conciencia fonémica: identificación de fonemas, con énfasis en el aislamiento de fonema inicial y final de palabras; emparejamiento de palabras por su fonema inicial y final.

Los ejercicios se presentaron en hojas de trabajo que contenían diferentes tareas, según el objetivo planteado para cada sesión. Las tareas combinaban estímulos visuales (dibujos) y auditivos. Las respuestas de los niños podían ser golpes de mano, respuestas verbales en vOz alta o marcas escritas en las hojas. Se emplearon sustantivos de carácter concreto o imaginable para facilitar su representación visual. Su longitud iba de 1 a 4 sílabas, y su estructura silábica también era variada (sílaba vocálica, directa, indirecta y con grupos consonánticos), aunque en los primeros ejercicios predominaban las más sencillas (V-CV-CVC). Con los niños de la escuela de párvulos, se enfatizó en conciencia léxica y silábica. Con los niños de primer año básico, se trabajaron las tres áreas por igual.

Al inicio de cada sesión, se reforzaba lo ejercitado anteriormente y luego se distribuía el nuevo material de trabajo. Una vez aplicado todo el programa, se procedió a reevaluar a los sujetos participantes del estudio con el mismo instrumento empleado en el postest.

\section{3.3. Procedimientos empleados en el análisis de los datos}

La primera fase del análisis consistió en la comparación de los puntajes T obtenidos en el pre y postest. Con el fin de comparar más detalladamente las diferencias en el propio desempeño, es decir, con independencia de los valores de referencia, en la segunda fase se analizaron los puntajes directos obtenidos por lo sujetos en cada subtest de la PSL.

Los datos se analizaron con el software estadístico SAS versión 9.1. Para las comparaciones entre pre y postest se aplicó el test $t$ de Student para muestras pareadas cuando las variables presentaban una distribución normal; en caso contrario, se empleó el test no paramérico de Wilcoxon. En todos los casos se utilizó un nivel de significancia de 0,05 .

\section{Análisis y discusión de resultados}

\subsection{Resultados obtenidos en el nivel transición 2}

El resumen de los resultados obtenidos por los niños de nivel transición 2 se presenta en la Tabla 2 (recuérdese que a este grupo solo correspondía evaluarle la 
conciencia silábica). Se muestra primero el puntaje total expresado como puntaje $\mathrm{T}$ y luego se señala el puntaje directo (PD) obtenido en las dos subpruebas que componen el test de conciencia silábica. En los dos casos, los paréntesis encierran las desviaciones típicas. La última columna indica el valor p alcanzado por la diferencia entre ambas mediciones.

Tabla 2. Resultados obtenidos en conciencia silábica por los niños de nivel transición 2 antes y después de la intervención.

\begin{tabular}{|l|c|c|c|c|c|}
\hline \multicolumn{1}{|c|}{ Prueba } & \multicolumn{2}{c|}{ Pretest } & \multicolumn{2}{c|}{ Postest } & Diferencia \\
\hline Conciencia silábica (T) & 41,5 & $(10,4)$ & 55,7 & $(5,4)$ & $<0,0001$ \\
\hline Segmentación silábica (PD) & 2,8 & $(2,6)$ & 6,8 & $(1,7)$ & 0,0002 \\
\hline Inclusión silábica (PD) & 0,5 & $(1,6)$ & 0,6 & $(1,0)$ & 0,3640 \\
\hline
\end{tabular}

De acuerdo con la norma empleada, el puntaje T inicial del grupo $(41,5)$ lo ubica justo en el límite inferior del desempeño típico acorde a su edad y NSE. Posteriormente, la comparación con el postest estableció que el incremento en el puntaje T alcanzado después de la intervención fue altamente significativo. En efecto, el grupo logró superar el promedio normativo (50), logrando un desempeño entre el promedio y el límite superior del desempeño típico esperado.

Al desagregar los datos para analizar los puntajes directos de ambos subtest, se observa que el incremento del puntaje $\mathrm{T}$ se explica por el mejoramiento significativo de la puntuación en la tarea de segmentación silábica. Es importante señalar que el promedio alcanzado en el postest iguala en segmentación silábica a los niños de nivel transición 2 con los de primer año básico al finalizar la intervención. Este aspecto de los resultados es promisorio, por la importancia que tiene el conocimiento de las sílabas en el desarrollo de las habilidades de descodificación de los niños prelectores.

También se observa que en ambas mediciones a los niños les resulta muy difícil incluir una sílaba en posición central de palabra: no logran promediar un ensayo correcto y mantienen el mismo bajo desempeño en el postest. Al respecto, se debe tener en consideración que la inclusión silábica es una tarea más compleja y corresponde a lo que Bravo Valdivieso et al. (2002) definen como 'tarea activa'. En efecto, la inclusión de una sílaba en posición central de palabra (ca[sa])ma) implica almacenar dos estímulos -la palabra y la sílaba que debe insertarse en ella- y, al mismo tiempo, manipularlos. Por lo tanto, resulta más demandante para la memoria de trabajo verbal y representa un estadio superior de desarrollo de la conciencia silábica que los niños de la muestra aún no alcanzan. En este sentido, los resultados obtenidos confirman que el desarrollo de la CF sigue un curso evolutivo y -al mismo tiempo-sugieren que la mejora en un aspecto no se puede generalizar a otros componentes. Esto también se ha encontrado en estudios con niños con algún retardo en el desarrollo del lenguaje y en niños con dificultades específicas de CF (Warrik \& Rowe-Walsh, 1993). 


\subsection{Resultados obtenidos en primer año básico}

En primer año básico, la evaluación de la CF mediante la PSL abarca tres niveles: conciencia léxica, silábica y fonémica. La Tabla 3 presenta los resultados del pre y postest expresados en puntajes T, y la diferencia entre ambas mediciones.

Tabla 3. Resultados obtenidos por los niños de 1er año básico antes y después de la intervención.

\begin{tabular}{|l|r|r|r|r|c|}
\hline Prueba & \multicolumn{2}{|c|}{ Pretest } & \multicolumn{2}{c|}{ Postest } & Diferencia \\
\hline Segmentación léxica & 49,4 & $(8,5)$ & 57,7 & $(9,2)$ & 0,0039 \\
\hline Conciencia silábica & 46,0 & $(12,2)$ & 55,7 & $(11,5)$ & 0,0007 \\
\hline Conciencia fonémica & 59,7 & $(15,3)$ & 66,1 & $(13,9)$ & 0,0167 \\
\hline
\end{tabular}

Como se puede observar, en segmentación léxica, el desempeño de los participantes de primer año básico en el pretest bordea los 50 puntos, con una puntuación acorde al promedio esperado para su edad y NSE. Al mismo tiempo, en segmentación silábica se evidencia un rendimiento grupal bajo los 50 puntos promedio, pero dentro de los límites considerados normales. Un aspecto de los resultados que llama la atención es el desempeño en segmentación fonémica, ya que en esta área los niños presentan el rendimiento inicial más alto, con un promedio que los ubica en el límite superior del desempeño esperado para su edad y NSE. Esto podría estar vinculado con la lectoescritura, ya que al momento de aplicar el pretest los alumnos habían comenzado su aprendizaje de la lengua escrita, por lo tanto, estaban empezando a familiarizarse con los fonemas.

En el postest, todos los puntajes suben de manera significativa, destacándose segmentación silábica. En el caso de la segmentación fonémica, el desempeño final del grupo lo ubica por sobre el desempeño promedio esperado para la edad y NSE.

Véase ahora el análisis de cada subtest, a fin de establecer las diferencias en cada una de las habilidades medidas. Recuérdese que los datos corresponden a los puntajes directos obtenidos por la muestra, lo que permite comparar el desempeño intragrupal antes y después de la intervención, independientemente de la norma. Los resultados se resumen en la siguiente tabla. 
Tabla 4. Puntajes directos obtenidos en pre y postest por escolares de primer año básico.

\begin{tabular}{|c|c|c|c|c|c|}
\hline Prueba & \multicolumn{2}{|c|}{ Pretest } & \multicolumn{2}{|c|}{ Postest } & Diferencia \\
\hline Segmentación léxica & 1,6 & $(1,7)$ & 3,4 & $(1,9)$ & 0,0038 \\
\hline \multicolumn{6}{|l|}{ Conciencia silábica } \\
\hline Análisis de sílabas & 4,8 & $(2,6)$ & 6,8 & $(1,9)$ & 0,0044 \\
\hline Inclusión de sílabas & 1,1 & $(1,9)$ & 2,1 & $(2,9)$ & 0,0004 \\
\hline \multicolumn{6}{|l|}{ Conciencia fonémica } \\
\hline Aislamiento fonema inicial & 2,0 & $(2,1)$ & 3,0 & $(1,8)$ & 0,0275 \\
\hline Análisis de fonemas & 1,1 & $(1,6)$ & 1,1 & $(1,6)$ & $>0,9999$ \\
\hline Síntesis de fonemas & 0,7 & $(1,2)$ & 1,8 & $(2,4)$ & $<0,0001$ \\
\hline
\end{tabular}

Tal como se indica en la Tabla 4, salvo en análisis de fonemas, en todas las demás áreas evaluadas los participantes subieron sus puntuaciones. En segmentación léxica, aumentó de manera significativa el número promedio de ensayos correctos, lo que quiere decir que mejoró la capacidad para percibir las palabras como unidades dentro del continum sonoro de la frase.

Se destaca el resultado alcanzado en conciencia silábica, ya que el rendimiento en el postest superó en las dos tareas los puntajes directos registrados en el pretest. La diferencia fue significativa en ambas tareas, especialmente en inclusión de sílaba, aun cuando los resultados confirman que esta última es una habilidad más compleja que la segmentación silábica.

En conciencia fonémica, al analizar las diferencias antes y después de la intervención, los resultados del postest revelan que hubo cambios significativos en las tareas de identificación del fonema inicial y en síntesis de fonemas. En el caso de esta última, la diferencia alcanzó un valor estadístico altamente significativo, lo que podría estar influido también por la ejercitación de la lectura, que involucra, en este nivel escolar, desarrollar la capacidad para unir los sonidos.

Por último, en contraste con lo señalado en el párrafo anterior, el promedio alcanzado por los participantes de primer año básico en el subtest de análisis de fonemas se mantuvo intacto en el postest. Al respecto, la literatura señala que la manipulación de fonemas se desarrolla más tardíamente que otras habilidades de CF y demanda mayor ejercitación. No obstante, tratándose de niños de primer año básico que ya habían iniciado su aprendizaje de la lengua escrita, los resultados sugieren la necesidad de seguir desarrollando intervenciones, ya que los antecedentes empíricos disponibles coinciden en que una vez iniciado el aprendizaje de la lectura el nivel de CF con mayor poder predictivo sobre el desempeño lector del niño es la conciencia fonémica. 


\section{Conclusiones finales}

El objetivo central del estudio presentado fue dar cuenta de los resultados obtenidos al comparar el antes y el después de una intervención destinada a desarrollar la CF en preescolares de nivel transición 2 y en escolares de primer año básico pertenecientes a establecimientos educacionales vulnerables. Aun cuando en esta oportunidad se informa de los resultados sin considerar grupo control, el análisis de la información recogida sobre el desempeño de los niños participantes evidencia efectos positivos y estadísticamente significativos del programa desarrollado. Vistos en conjunto, los resultados permiten señalar que el aspecto de la CF que más se benefició con la intervención llevada a cabo fue el nivel silábico, seguido por la conciencia léxica y, finalmente, por la conciencia fonémica. Los resultados, por lo tanto, son concordantes con lo que señala la literatura en cuanto a que la sílaba es la unidad que los niños aprenden más fácilmente a manejar.

El logro demostrado en conciencia silábica por los participantes de la escuela de párvulos, que ubica al grupo en el rango superior del desempeño típico para la edad y NSE ( $\mathrm{T}=55,7)$, muestra que las actividades realizadas en esta área durante la implementación del programa resultaron efectivas para el desarrollo de la habilidad de segmentación silábica en estos niños. Escuchar palabras y reconocer las sílabas que las conforman es un buen predictor del desempeño en las primeras etapas del aprendizaje del código escrito. En este sentido, los resultados sugieren un avance positivo en vistas al inicio del aprendizaje de la lectura de los niños de nivel transición 2 que participaron del estudio.

En relación con los resultados obtenidos por el mismo grupo en inclusión silábica, que corresponde a la segunda tarea de conciencia silábica evaluada por la PSL, estos confirman que se trata de una tarea más demandante que la de segmentación, y que resulta muy compleja para los niños prelectores, tanto en el pretest como en el postest. Los resultados son coincidentes con los conseguidos en investigaciones como la de Arnáiz et al. (2002), en la que luego de implementar durante dos años un programa de entrenamiento de la CF en niños de 5 y 6 años, el grupo intervenido mejoró sus habilidades de manipulación silábica (vg. inversión de sílaba, omisión de sílaba medial), pero estas se mantuvieron por debajo del desempeño demostrado en las tareas menos complejas como el reconocimiento y la segmentación de sílabas.

Al mismo tiempo, la información recogida a través de ambas mediciones, así como el análisis de la experiencia por parte de las investigadoras, permiten concluir que es necesaria una intervención más prolongada o sistemática para que los niños prelectores logren manipular de manera más activa las sílabas que conforman las unidades léxicas.

En primer año básico, mejoró de manera significativa el rendimiento en los niveles léxico y silábico. En conciencia silábica, los niños aumentaron los puntajes en los 
dos subtest, lo que sugiere un papel importante del nivel escolar en el desarrollo de la capacidad para manipular activamente las sílabas. En el nivel fonémico, hubo una mejoría significativa en el reconocimiento del fonema inicial y en síntesis de fonemas, pero se mantuvo el bajo desempeño en análisis fonémico. Este tipo de tarea también ha mostrado ser más resistente en otras investigaciones.

En su conjunto, los resultados conseguidos en conciencia fonémica por los niños de primer año básico son concordantes con la idea de que la instrucción lectora beneficia su desarrollo, dado que aprender a leer implica una atención especial a segmentos lingüísticos más pequeños que la sílaba para poder establecer el correlato fonológico de los grafemas. A su vez, la investigación existente en el área da cuenta de que un buen desempeño en conciencia fonémica facilita la lectura, pues beneficia los procesos de descodificación. En este sentido, una limitación del trabajo realizado es no haber evaluado la lectura de los niños de primer año básico al inicio. Por lo tanto, en investigaciones futuras se deberá incluir una evaluación de la lectura de los participantes que permita precisar los alcances de la relación causal mutua entre conciencia fonológica y lectura, antes y después de ser aplicado un programa de entrenamiento.

Otro aspecto que habría que considerar a futuro es el control de variables instruccionales relacionadas con los métodos de enseñanza de la lectoescritura que aplica la escuela. Procedimientos cualitativos como entrevistas al profesor, revisión de los materiales que se emplean para el aprendizaje de la lectura y la observación de clases permiten recoger información que puede resultar muy valiosa a la hora de aplicar un programa de entrenamiento en CF y evaluar sus efectos en los distintos niveles de esta así como en el aprendizaje de la lectura propiamente tal.

En relación con los materiales diseñados, probablemente la incorporación de actividades como el reconocimiento de letras y la escritura de palabras habría facilitado las tareas más complejas tanto a nivel silábico como fonémico. Los antecedentes disponibles indican que el trabajo combinado con la presentación visual de letras y palabras, y la escritura al dictado de palabras favorece el desarrollo de la conciencia fonémica (Arnáiz et al., 2002; Cuadro \& Trías, 2008).

El trabajo que hemos presentado se proyecta basado en un enfoque de prevención de posibles dificultades en el aprendizaje de la lectura. Por lo mismo, las tareas diseñadas se han concebido principalmente para su aplicación grupal, ya sea a todo el grupo curso o a pequeños grupos. Sin embargo, también se podrían utilizar individualmente con niños que presenten algún retraso en su aprendizaje de la lectura, con la debida revisión por parte del profesional correspondiente (psicopedagogos, educadores diferenciales) para implementar posibles adaptaciones a las necesidades específicas del niño. 
Se ha hablado hasta aquí principalmente de actividades. No obstante, un programa de entrenamiento de la CF no se puede limitar a un conjunto de tareas. Un programa de entrenamiento en $\mathrm{CF}$ se tiene que hacer cargo tanto de los procesos como de los sujetos y los contextos en que ellos interactúan. Por lo tanto, se debe considerar el papel de la motivación, la sensibilización del niño hacia los estímulos auditivos, las características del entorno de aprendizaje, el rol de los adultos, especialmente el papel mediador que cumple el adulto responsable del aula. Un programa debe considerar también la evaluación, pero no solo al comienzo y final, sino el monitoreo o evaluación de los logros que van alcanzando los niños durante el proceso. Por último, recoger la opinión de educadoras, profesores y psicopedagogos podría ser otra alternativa de validación del programa que podría resultar interesante. Todo lo señalado no es sino un resumen de los desafíos pendientes que deberá enfrentar el equipo de investigación.

Por último, a pesar de sus limitaciones, la experiencia llevada a cabo muestra que es posible incidir tempranamente en el desarrollo de la CF en contextos educativos de alta vulnerabilidad social, lo que invita a seguir desarrollando investigaciones de campo que contribuyan a generar condiciones de mayor equidad en el acceso a la cultura letrada. 


\section{REFERENCIAS BIBLIOGRÁFICAS}

Anthony, J. \& Francis, D. (2005). Development of phonological awareness. Current Directions in Psychological Science, 14(5), 255-259.

Arnáiz, S., Castejón, J., Ruiz, M. \& Guirao, J. (2002). Desarrollo de un programa de habilidades fonológicas y su implicación en el acceso inicial a la lectoescritura en alumnos de segundo ciclo de educación infantil. Revista Educación, Desarrollo y Diversidad, 5(1), 29-51.

Berdicewski, O. \& Milicic, N. (2002). Prueba de Funciones Básicas. $15^{a}$ Edición. Santiago: Editorial Universitaria.

Bizama, M., Arancibia, B. \& Sáez, K. (2011). Evaluación de la conciencia fonológica en párvulos de nivel transición 2 y escolares de primer año básico, pertenecientes a escuelas vulnerables de la Provincia de Concepción, Chile. ONOMAZEIN, 23(1), 81-103.

Bravo Valdivieso, L. (2002). La conciencia fonológica como una zona de desarrollo próximo para el aprendizaje inicial de la lectura. Revista Estudios Pedagógicos, $28,165-177$.

Bravo Valdivieso, L. (2006). Lectura inicial y psicología cognitiva. Santiago: Ediciones Pontificia Universidad Católica.

Bravo Valdivieso, L., Villalón, M. \& Orellana, E. (2001). Procesos predictivos del aprendizaje inicial de la lectura en primero básico. Boletín de Investigación Educacional, 16, 149-160.

Bravo Valdivieso, L., Villalón, M. \& Orellana, E. (2002). La conciencia fonológica y la lectura inicial en niños que ingresan a primer año básico. Psykhé, 11, 175182.

Bravo Valdivieso, L., Villalón, M. \& Orellana, E. (2003a). Diferencias de preparación para el aprendizaje de la lectura en niños que ingresan al primer año básico. Boletín de Investigación Educacional, 16, 156-171.

Bravo Valdivieso, L., Villalón, M. \& Orellana, E. (2003b). Predictividad del rendimiento de la lectura: El segundo año básico. Psykhé, 12, 29-36.

Bravo Valdivieso, L., Villalón, M. \& Orellana, E. (2006). Predictibilidad del rendimiento en la lectura: Una investigación de seguimiento entre primer y tercer año. Revista Latinoamericana de Psicología, 38, 1-20.

Carrillo, M. S. (1994). Development of phonological awareness and reading acquisition. A study in Spanish language. Reading and Writing: An Interdisciplinary Journal, 6, 279-298. 
Carrillo, M. S. \& Sánchez, J. (1991). Segmentación fonológico-silábica y adquisición de la lectura: Un estudio empírico. Comunicación, Lenguaje y Educación, 9, 109116.

Carrillo, M. S. \& Marín, J. (1996). Desarrollo metafonológico y adquisición de la lectura: Un programa de entrenamiento. Madrid: Servicio de publicaciones MEC.

Cuadro, A. \& Trías, D. (2008). Desarrollo de la conciencia fonémica: Evaluación de un programa de intervención. Revista Argentina de Neuropsicologia, 11, 1-8.

De la Torre, C., Guerrero, D., Conde, I. \& Claros, R. (2002). Komunica. Programa para el desarrollo del conocimiento fonológico. Málaga: Aljibe.

Fox, B. \& Routh, D. (1975). Analyzing spoken language into words, syllables and phonemes: A developmental study. Journal of Psycholinguistic Research, 4, 331-342.

Fundación Astoreca (2007). Manual de Lenguaje kínder [en línea]. Disponible en: http://www.educandojuntos.cl/dms/doc_3658.html

Gathercole, S. \& Baddeley, A. (1993). Working memory and language. Nueva York: Psychology Press.

Gillam, R. \& van Kleeck, A. (1996). Phonological awareness training and short-term working memory: Clinical implications. Topics in Language Disorders, 17, 7281.

Herrera, L. \& Defior, S. (2005). Una aproximación al procesamiento fonológico de los niños prelectores: Conciencia fonológica, memoria verbal a corto plazo y denominación. Psykhé, 14(2), 81-95.

Hulme, Ch., Hatcher, P., Nation, K., Brown, A., Adams, J. \& Stuart, G. (2002). Phoneme awareness is a better predictor of early reading skill than onsetrime awareness. Journal of Experimental Child Psychology, 8, 2-28.

Jiménez, J. (1992). Metaconocimiento fonológico: Un estudio descriptivo sobre una muestra de niños prelectores en edad escolar. Infancia y Aprendizaje, 57, 4966.

Jiménez, J. (1996). Conciencia fonológica y retraso lector en una ortografía transparente. Infancia y Aprendizaje, 76, 109-121.

Jiménez, J. (2009). Manual para la evaluación inicial de la lectura en niños de educación primaria. [en línea]. Disponible en: http://www.ineesite.org/toolkit/INEEcms/uploads/1089/Early_Grade_Reading_Assessment_Toolkit_SP.pdf 
Jiménez, J. \& Ortiz, M. (1993). Phonological awaraness in learning literacy. Cognitiva, $1,3-27$.

Jiménez, J. \& Ortiz, M. (1995). Conciencia fonológica y aprendizaje de la lectura: Teoría, evaluación e intervención. Madrid: Síntesis.

Jiménez, J. \& Ortiz, M. (2000). Conciencia metalingüística y adquisición lectora en la lengua española. The Spanish Journal of Psychology, 3(1), 36-46.

Jiménez, J. \& Ortiz, M. (2007). Conciencia fonológica y aprendizaje de la lectura: Teoría, evaluación e intervención. Madrid: Síntesis.

Jiménez, J., García, E. \& Venegas, E. (2010). Are phonological processes the same or different in low literacy adults and children with or without reading disabilities in a consistent orthography? Reading and Writing: An Interdisciplinary Journal, 23, 1-18.

Liberman, I., Shankweiler, D., Fischer, W. \& Carter, B. (1974). Explicit syllable and phoneme segmentation in the young child. Journal of Experimental Child Psychology, 18, 201-212.

Mann, V. \& Liberman, I. (1984). Phonological awareness and verbal short-term memory. Journal of Learning Disabilities, 17, 592-599.

Manrique, A. \& Gramigna, S. (1984). La segmentación fonológica y silábica en niños de preescolar y primer grado. Lectura y Vida, 5(1), 4-13.

Márquez, J. \& de la Osa, P. (2003). Evaluación de la conciencia fonológica en el inicio lector. Anuario de Psicología, 34(3), 357-370.

McCallum, R., Bell, S., Wood, M., Below, J., Choate, S. \& McCane, S. (2006). What is the role of working memory in reading relative to the big three processing variables (orthography, phonology, and rapid naming)? Journal of Psychoeducational Assessment, 24(3), 243-259.

Orellana, E. \& Ramaciotti, A. (2007). Prueba de segmentación lingüística. Santiago: Ediciones Pontificia Universidad Católica.

Pavez, M., Maggiolo, M., Peñaloza, C. \& Coloma, C. J. (2009). Desarrollo fonológico en niños de 3 a 6 años: Incidencia de la edad, el género y el nivel socioeconómico. Revista de Lingüistica Teórica y Aplicada, 47(2), 89-109.

Porta, M. (2008). Comprendiendo el rol predictivo de la conciencia fonológica en la adquisición de la lectura y en la detección de niños en riesgo pre-lector. Revista de Orientación Educacional, 22(42), 87-103. 
Rincón, M. \& Pérez, J. (2009). Programa para el entrenamiento de la conciencia fonológica en niños de 5 a 7 años como prerrequisito para el aprendizaje de la lectura. Revista Areté, 9, 140-150.

Rueda, M. (1995). La lectura: Adquisición, dificultades e intervención. Salamanca: Amarú Ediciones.

Rueda, M., Sánchez, E. \& González, L. (1990). El análisis de la palabra como instrumento para la rehabilitación de la dislexia. Infancia y Aprendizaje, 49, $39-52$.

Scanlon, D. \& Vellutino, F. (1997). A comparison of the instructional backgrounds and cognitive profiles of poor, average, and good readers who were initially identified as at risk for reading failure. Scientific Studies of Reading, 1, 191-216.

Velarde, E. (2010). Aplicación de un programa metafonológico en niños(as) de 8 a 10 años. Investigación Educativa, 14(25), 137-154.

Vigotzky, L. (1988). El desarrollo de los procesos psicológicos superiores. Madrid: Editorial Crítica Visor.

Treiman, R. (1991). Phonological awareness and its roles in learning to read and spell. En D. Sawyer \& B. Fox (Eds.), Phonological Awareness in Reading: The Evolution of Current Perspective (pp. 1-30). Nueva York: Springer-Verlag. Warrik, N. \& Rowe-Walsh, S. (1993). Phoneme awareness in language delayed children: Comparative studies and intervention. Annals of Dyslexia, 43, 153-173.

\section{NOTAS}

1. Investigación Código DIN-11-2009, patrocinada por la Dirección de Investigación, Universidad Católica de la Santísima Concepción (UCSC).

2. Véase resultados SIMCE http://www.simce.cl/index.php?id=122\&no_cache=1 